\title{
Correction to: High phase space density loading of a falling magnetic
} trap

\author{
Ido Almog ${ }^{1} \cdot$ Jonathan Coslovsky $^{1} \cdot$ Gil Loewenthal $^{1} \cdot$ Arnaud Courvoisier $^{1}{ }^{\mathbb{C}} \cdot$ Nir Davidson $^{1}$
}

Published online: 13 August 2018

○) Springer-Verlag GmbH Germany, part of Springer Nature 2018

\section{Correction to: Applied Physics B (2018) 124:158}

\section{https://doi.org/10.1007/s00340-018-7025-7}

In the original publication of the article, typesetters have incorrectly processed the Eq. 2 . The correct equation should read as given below:

$\frac{\mathrm{d} \log \left(b_{z}\right)}{\mathrm{d} t}(t)=\gamma \tau_{0}^{-1}\left(\frac{b_{z}(t)}{b_{0}}\right)^{2 / 3}$

The original article has been corrected.

1 Department of Physics of Complex Systems, Weizmann Institute of Science, 76100 Rehovot, Israel 